\title{
Applying Bubbles to Localize Features That Control Pigeons' Visual Discrimination Behavior
}

\author{
Brett M. Gibson and Edward A. Wasserman \\ The University of Iowa
}

\author{
Frédéric Gosselin \\ Université de Montréal
}

\author{
Philippe G. Schyns \\ University of Glasgow
}

\begin{abstract}
The authors trained pigeons to discriminate images of human faces that displayed: (a) a happy or a neutral expression or (b) a man or a woman. After training the pigeons, the authors used a new procedure called Bubbles to pinpoint the features of the faces that were used to make these discriminations. Bubbles revealed that the features used to discriminate happy from neutral faces were different from those used to discriminate male from female faces. Furthermore, the features that pigeons used to make each of these discriminations overlapped those used by human observers in a companion study (F. Gosselin \& P.G. Schyns, 2001). These results show that the Bubbles technique can be effectively applied to nonhuman animals to isolate the functional features of complex visual stimuli.
\end{abstract}

Keywords: bubbles, visual discrimination, categorical learning, stimulus control, face recognition
For many years, researchers have sought to understand how nonhuman animals learn to categorize objects (for a review, see Herrnstein, 1990), that is, how nonhuman animals come to treat discriminably different objects or events as members of a common class and respond similarly to them. Researchers have discovered that animals readily categorize objects according to their perceptual similarity (Bhatt, Wasserman, Reynolds, \& Knauss, 1988; Herrnstein, Loveland, \& Cable, 1976; Lea, Lohmann, \& Ryan, 1993). Such perceptual category learning is an important process that allows animals to respond flexibly to a variable environment. Although category learning has been well documented in the nonhuman animal literature, investigators of category learning have encountered a vexing dilemma.

One the one hand, researchers who examine category learning often use complex visual stimuli to document the ability of nonhuman animals to form categories that comprise highly heterogeneous members (e.g., Herrnstein et al., 1976; Jitsumori \& Yoshihara, 1997); such usage enhances the verisimilitude of the experimental stimuli. The results of these studies may be especially interesting because the discriminative stimuli in animals' natural habitats are usually far more complex than the artificial discriminative stimuli conventionally given in laboratory studies

Brett M. Gibson and Edward A. Wasserman, Department of Psychology, The University of Iowa; Frédéric Gosselin, Département de psychologie, Université de Montréal, Montréal, Quebec, Canada; Philippe G. Schyns, Department of Psychology, University of Glasgow, Glasgow, Scotland, United Kingdom.

We thank Ludwig Huber for his helpful comments concerning this article.

Correspondence concerning this article should be addressed to Brett M. Gibson, who is now at the Department of Psychology, Conant Hall, 10 Library Way, Durham, NH 03824. E-mail: bgibson@cisunix@unh.edu of learning. On the other hand, an important limitation of using complex naturalistic stimuli in studies of visual discrimination learning is that it is extremely difficult to isolate the features of the stimuli that are truly diagnostic (von Ferson \& Lea, 1990) of the reinforcement contingencies and that effectively control animals' discriminative responding. Indeed, this limitation has prompted researchers to use simple artificial stimuli to better isolate the perceptual mechanisms of category discrimination (e.g., Huber \& Lenz, 1993; Lea et al., 1993; Mackintosh, 1995; Wasserman, 1993).

Although the use of simplified stimuli in category learning experiments has been informative, the way that animals classify simple artificial and complex naturalistic stimuli might be quite different. Therefore, it would be extremely helpful to have an investigative tool that allowed researchers to use complex visual stimuli in the psychological laboratory while still allowing them to isolate the features of the stimuli that control discriminative behavior.

Recently, a new procedure called Bubbles (Gosselin \& Schyns, 2001, 2002, 2004; Schyns, Bonnar, \& Gosselin, 2002) was developed to isolate the local features of visual stimuli that are used by human participants during categorization. In an initial study, Gosselin and Schyns (2001) trained human participants to discriminate photographs of faces of eight adult men and eight adult women that displayed either a happy or a neutral expression (see Figure 1a). Each participant was required to discriminatively report the identity of the face, whether the face was that of a man or a woman. During testing, the participant encountered the same faces that were given during training, but this time each face was covered by a midgray mask (see Figure $1 b$ ) that revealed only a portion of the underlying face through openings or "bubbles" in the mask. The facial features that were revealed though the mask were deemed useful for making the discrimination on one of the dimensions selected by the experimenter if the participant could 
a
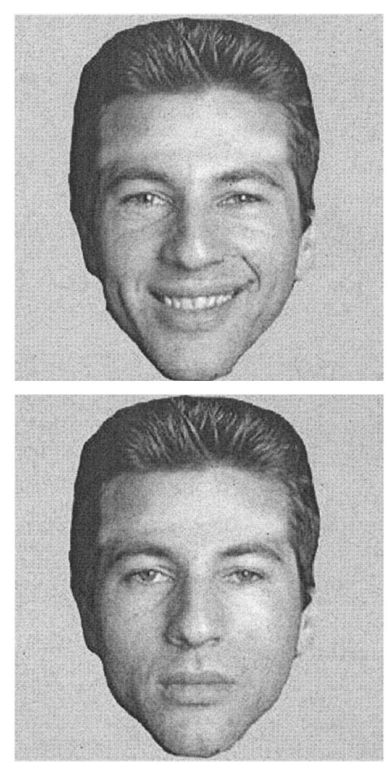

b
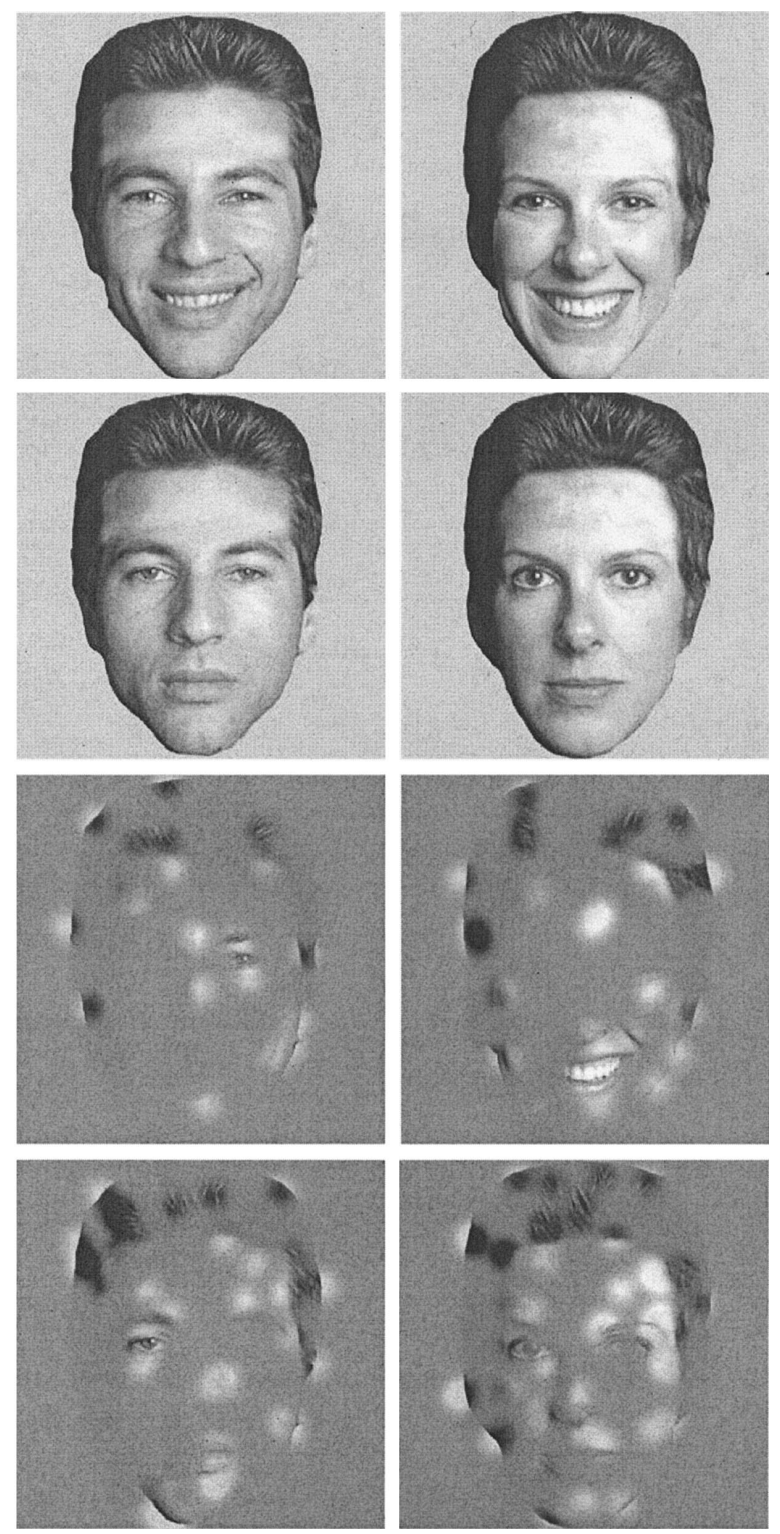

Figure 1. Examples of the images used in the present experiment. (a) Four of the 32 images used in training, which show one of the eight men (left column) and one of the eight women (right column) exhibiting either a happy (top row) or a neutral (bottom row) expression. (b) Examples of 4 bubbled faces used in testing, which show testing displays with 20 bubbles (Phase 1: top left), 30 bubbles (Phase 2: top right), 40 bubbles (Phase 3: bottom left), and 50 bubbles (Phase 4: bottom right).

still successfully discriminate the stimulus. The position of the openings in the mask varied randomly across trials, so that all areas of the face were equally likely to be revealed and the most important features could be isolated.

The Bubbles procedure indicated that human participants used features around the mouth (see Figure $2 b$ ) to discriminate happy from neutral expressions (emotion discrimination), and they used features around the eyes and mouth to discriminate men from women (gender discrimination). Although the Bubbles procedure has only been used with human participants, it might also be an effective method for examining the features of complex stimuli that nonhuman animals use when making visual discriminations.

In the present study, we administered the Bubbles procedure for the first time to nonhuman animals to assess its usefulness in isolating the features of complex stimuli that control the discriminative behavior of a nonhuman animal. We used human faces as stimuli because (a) they contain a wealth of visual information, (b) they can be discriminated along multiple dimensions (e.g., gender, expression, identity), (c) they are complex naturalistic stimuli, and (d) they have been used successfully in prior applications of the Bubbles procedure with human participants. We selected pigeons as experimental animals because (a) they have excellent vision, (b) we know a great deal about arranging effective reinforcement contingencies to conduct sensitive psychophysical testing procedures, and (c) there is little reason to suspect that they have individual or evolutionary dispositions to discriminate human faces.

A final feature of the present study is its possible comparative significance. If the Bubbles procedure can be effectively used with nonhuman animals, then the features of stimuli that are functional for different species can be directly compared with one another. Similar features might suggest the participation of similar perceptual processes; different features might suggest the participation of different perceptual processes.

\section{Method}

\section{Subjects}

Four adult feral pigeons (Columba livia) that were maintained at $85 \%$ of their ad lib weights were used in this study. All of the pigeons were given controlled feedings of mixed grain and had free access to water that contained a vitamin supplement. The pigeons had participated in an unrelated project in which they were presented geometrical shapes on a computer screen (Gibson \& Wasserman, 2003) prior to participating in the current experiment.

\section{Apparatus}

Each pigeon was trained and tested in one of four operant conditioning chambers. Visual images were shown in the center of a computer monitor (15 in.; $38.1 \mathrm{~cm})$, and responses were recorded from red and green circular report areas (1.9-cm diameter) that were located to the northwest and to the northeast of the image, respectively. A touch screen recorded the location of responses (pecks) that the birds made to the screen. A rotary feeder dispensed 45-mg food pellets into a food cup inside the chamber.

\section{Procedure}

Discrimination training. Each pigeon was trained to discriminate 32 gray scale photographs of the faces of eight men and eight women professional actors exhibiting either happy or neutral expressions (Figure 1a). The full-face images $(7 \mathrm{~cm} \times 7 \mathrm{~cm})$ were professionally photographed with controlled illumination from a light source that was positioned on the right and top of the face (Oliva \& Schyns, 2000). The images had normalized hairstyles, and both the position of the face on the image and the size of the face were normalized. The images were identical to those used in an initial test of the Bubbles procedure with human participants (Gosselin \& Schyns, 2001).

A trial began when a black cross stimulus appeared in the display area; a single peck to the display area advanced the trial. Next, 1 of the 32 face photographs was randomly selected and appeared in the center of the 
Humans
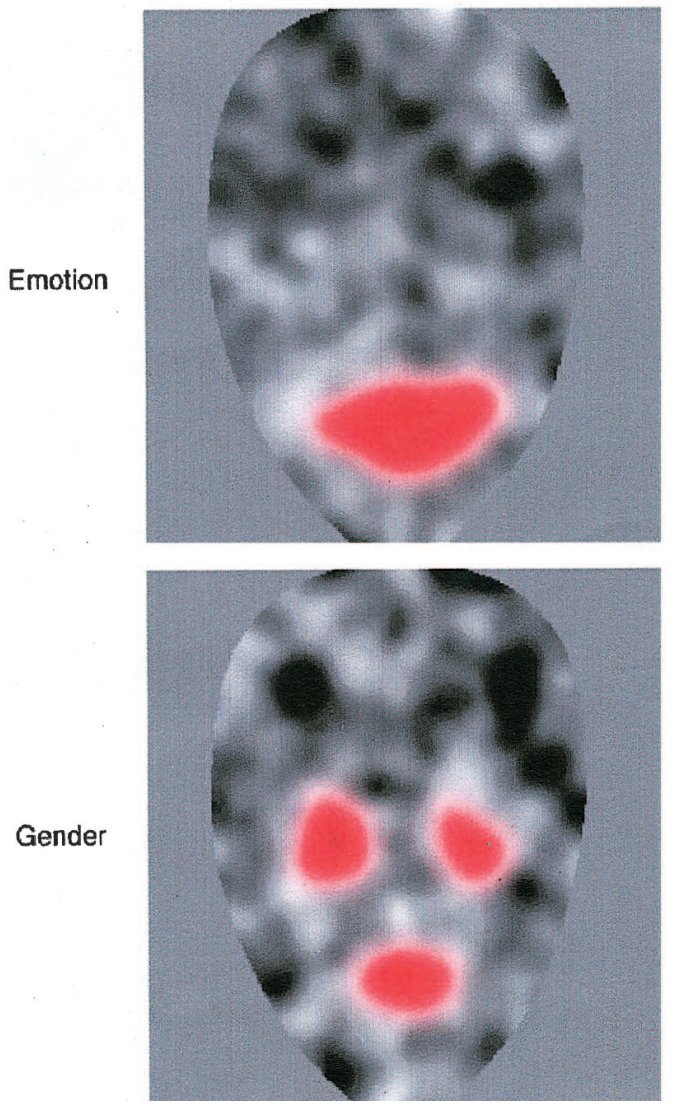

Pigeons
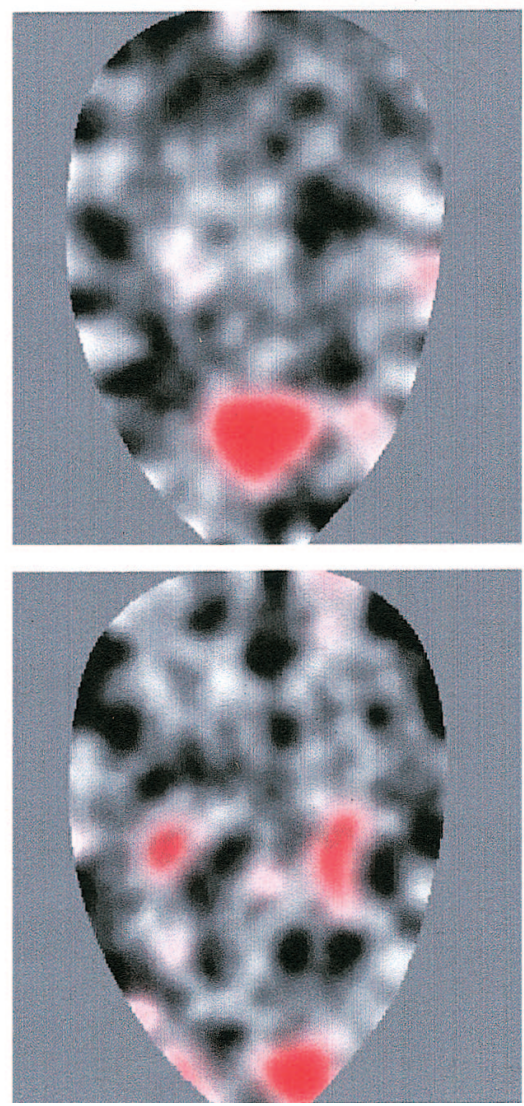

Ideals
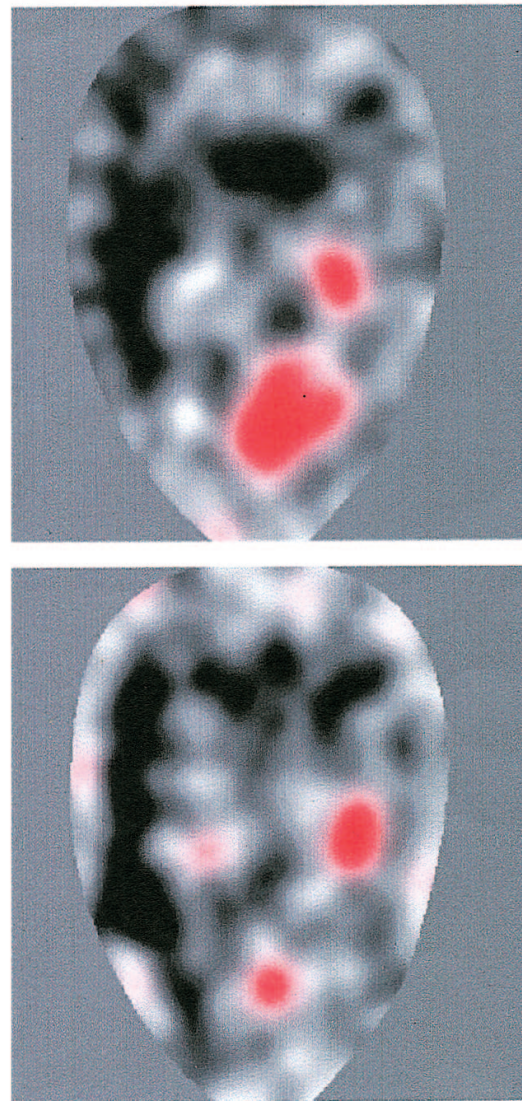

Figure 2. Composite diagnostic masks following Bubbles testing and analysis for humans, pigeons, and ideal observers on the gender (top row) and emotion (bottom row) discrimination tasks. Each diagnostic mask is placed over the same female face for illustrative purposes only. Input areas that were used reliably above chance are shown in red and reveal the underlying face below. Regions of the input space that were used no more than chance are colored gray, and those that were used below chance are colored black (see Method section).

monitor. The pigeon was then required to make a fixed number of observing responses (at any location) to the image, after which the red and green choice areas were illuminated. One pair of pigeons (31B, 23Y) was trained to discriminate whether the face displayed a happy or a neutral expression (emotion condition), whereas a second pair of pigeons $(52 \mathrm{~W}, 72 \mathrm{Y})$ was trained to discriminate whether the face was a man's or a woman's (gender condition). A correct choice resulted in the delivery of a food pellet, the termination of the display, and the clearing of response areas from the screen. Following an incorrect choice, the trial was repeated until a correct response was made (the color of the correct report area was counterbalanced across birds in each condition). An intertrial interval with a mean of $10 \mathrm{~s}$ (range $=5-15 \mathrm{~s}$ ) followed the termination of each trial. Each block of training contained 32 trials (one for each of the faces); five blocks of training were conducted per daily session for a total of 160 trials. Training continued until the birds were averaging $70 \%$ correct responses to each of the four stimulus types (male-neutral, male-happy, female-neutral, female-happy). Training lasted 60 days for pigeon 31B, 98 days for pigeon $23 \mathrm{Y}$ (emotion condition), 165 days for pigeon $52 \mathrm{~W}$, and 78 days for pigeon $72 Y$ (gender condition).

Bubbles testing. During testing, we presented the birds with the same 32 faces they had encountered during training, but this time each image was only partly visible through a midgray mask (Figure 1b); the overlying mask was punctured by several small Gaussian windows or bubbles $(S D=$
$0.27 \mathrm{~cm}$ in diameter) that revealed portions of the face below. The bubbles were randomly positioned over each face across trials so that each bubbled testing stimulus was unique. Across blocks of extended testing, the entire face was disclosed and the facial features that were revealed were unbiased. The other events that occurred during the trial were identical to those described for training.

During each block of testing, the pigeons were presented with the 32 training faces plus 2 bubbled testing faces from each of the four different stimulus categories (Figure 1b). One five-block session of testing was administered each day. The pigeons continued with testing across consecutive days if performance to the four types of training images (maleneutral, male-happy, female-neutral, female-happy) during testing remained above $70 \%$ correct; otherwise, they were returned to training until they met this performance criterion. We tested the birds in seven consecutive phases with approximately 320 bubbled images in each phase (40 testing images per session $\times 8$ sessions). The performance of human participants has typically been maintained between baseline and ceiling levels of performance during Bubbles testing by titrating the number and size of the bubbles in the mask (Gosselin \& Schyns, 2001, 2002). However, in the current experiment, the number of bubbles per stimulus was systematically varied across the seven experimental phases; successive testing phases involved stimuli with 20, 30, 40, 50, 40, 30, and 20 bubbles, respectively. 


\section{Bubbles Analysis}

Following the conclusion of the experiment, we used special analytical procedures (see Gosselin \& Schyns, 2001, 2002, 2004) to pinpoint the features of the faces that the pigeons used to discriminate both human emotion and gender. The location of the bubbles in a mask changed randomly across trials, thereby revealing different features of the face. On some trials, the facial features revealed through the mask may have contained enough information to support a correct choice (e.g., to discern whether a face was that of a man or a woman), but on other trials, the mask may not have revealed enough information to support a correct response. For example, it may be relatively difficult to discern whether the face in the upper left corner of Figure $1 \mathrm{~b}$ is happy or neutral, but it may be relatively easy to do so for the face in the upper right corner.

We used the pigeons' choices when they were presented with the bubbled images to ascertain which features of the faces controlled their discriminative response. Specifically, each face comprised a matrix of $256 \times 256$ pixels $(65,536$ total pixels $)$ On any given trial, the bubbles revealed some of the face pixels beneath, whereas most of the face pixels remained hidden. We generated a ratio score for each pixel for each of the 32 faces, which specified the probability that the pigeon responded correctly when a particular pixel was revealed by a bubble. For example, on one trial, a pixel on the mouth of a male-happy face might be visible through a bubble. If the pigeon responded correctly to that testing image (e.g., for the gender discrimination, a correct response indicated that the face was a male), then the ratio score for that pixel would be $1 / 1$, or $100 \%$ (the same would be true for all of the other pixels of the face that were revealed on that trial). If, on the next trial, the same pixel of the face was again revealed, but this time the pigeon responded that the face was female, then the ratio score would be adjusted to one half, or $50 \%$ for that particular pixel. A high ratio score tended to indicate that a pixel was part of a feature or region that was more diagnostic, whereas a low ratio score tended to indicate that a particular pixel was part of a feature or region that was less diagnostic. The mean ratio score for each pixel was transformed into a $Z$ score. The sum of all of the $Z$ scores per pigeon per task was $Z$ scored, which yielded a set of summary $Z$ scores for the two birds in each task.

$\mathrm{Z}$ score summary images. We then constructed a diagnostic display of the summary $Z$ scores for the gender and emotion tasks. The $Z$ scores for each pixel of each summary were associated with a gray scale shade, so that pixels whose scores were above chance $(Z>1.65, p<.05)$ were clear (colored red for diagnostic purposes in Figure 2) and revealed a portion of the image beneath. Regions of the input space that were used no more than chance were colored gray, and regions of the input space that were reliably below chance were colored black.

Human and ideal observers. We then performed a correlation that examined the overlap in the use of facial features for each task by pigeons in the current study with humans' and ideal observers' (Tjan, Braje, Legge, \& Kersten, 1995) use of facial features in a companion study (Gosselin \& Schyns, 2001). The ideal observer provided a benchmark of the information available in the stimulus set to solve each task. The ideal observer is a computer that had complete information regarding the 32 faces and was programmed to capture all of the regions of the image that had the highest local variance between the considered categories (male vs. female and neutral vs. emotional). The ideal observer encountered Bubbles testing, just like our biological participants, and it used the information available in the bubbled image to determine to which category the test stimulus belonged. The ideal observer considers the stimuli as images (not faces composed of eyes, a nose, and a mouth, as humans do), and it need not be sensitive to the regions that human and nonhuman animals find most useful (the diagnostic regions), but rather can be sensitive to the information that is available in the data set for the task at hand. The choices of the ideal observer were then used to calculate $Z$-scored coefficient maps as explained above. This observer was ideal because it had complete knowledge of the stimulus set, and it used this information during testing to make the fewest number of errors on average. Hence, the ideal observer provides the standard for the information that was available in the stimulus set for a given discrimination.

\section{Results}

Accuracy scores on the unaltered training stimuli averaged across all seven phases of testing were $88 \%$ (31B) and $78 \%(23 \mathrm{Y})$ for the 2 pigeons in the emotion condition and $70 \%(52 \mathrm{~W})$ and $78 \%$ (72Y) for the two birds in the gender condition. Percentage correct choices on the testing displays were somewhat lower. Accuracy scores on the testing stimuli were kept low through the use of fewer bubbles to maximize the sensitivity of the Bubbles procedure. If too many bubbles are used, then the ratio scores of individual pixels increase broadly and a larger percentage of the pixels of the stimulus are deemed to be diagnostic; with humans, performance is generally maintained around $75 \%$ by titration (Gosselin \& Schyns, 2001).

The 2 pigeons in the emotion condition averaged 54\%, 56\%, $60 \%, 65 \%, 60 \%, 57 \%$, and $57 \%$ correct choices across each of the seven phases $(20,30,40,50,40,30$, and 20 bubbles, respectively) of the experiment, whereas the 2 pigeons in the gender condition averaged $51 \%, 52 \%, 57 \%, 60 \%, 60 \%, 57 \%$, and $54 \%$ correct choices, respectively, during the same period. Accuracy was generally higher during the second round of testing with the 20,30, and 40 bubbles stimuli than was the case during the first three sets, perhaps because the pigeons were learning further during the testing trials.

The accuracy scores of pigeons in the emotion condition tended to be somewhat higher than the accuracy scores of pigeons in the gender condition, which is consistent with the difference in the speed of the pigeons' discrimination learning. Likewise, human subjects in a companion study also had more difficulty mastering the gender task than the emotion task (Gosselin \& Schyns, 2001). Accuracy to the testing stimuli progressively improved with increasing numbers of bubbles. Strong correlations were observed between the number of bubbles and choice accuracy for each bird (emotion condition, $r_{31 \mathrm{~B}}=.91$ and $r_{23 \mathrm{Y}}=.75$; gender condition, $r_{52 \mathrm{~W}}=0.71$ and $\left.r_{72 \mathrm{Y}}=.85\right)$.

For the Bubbles analysis, we analyzed only those phases in which choice accuracy was significantly above chance $(p<.05$, binomial tests). For the emotion task, 11 of 14 phases met the criterion (4 sets for $23 \mathrm{Y}$; 7 sets for $31 \mathrm{~B}$ ), and for the gender task, 8 of 14 phases met the criterion ( 4 sets for $52 \mathrm{~W}$; 4 sets for $72 \mathrm{Y}$ ) and were included in the analysis.

Figure 2 presents the results from the bubbles analysis. It displays the full wealth of facial features that were used by pigeons in the current project and by humans and ideal observers in a companion project (Gosselin \& Schyns, 2001). The use of features by the pigeons was decidedly nonrandom; rather, the birds used features of the face that varied with the task they were required to perform. Pigeons that were discriminating between happy and neutral expressions used features in the bottom part of the face including the mouth, whereas pigeons that were discriminating between male and female faces used regions near the eyes and chin. The areas of the face that each pigeon in each condition used overlapped extensively (data not shown). The ideal observer indicated that regions on the left side of the face were more informa- 
tive than those on the right, perhaps because the light source for the face photographs was positioned to the right of the face, and the shading on the left increased the contrast information.

Notably, the features of the face that were used by our pigeons correlated with those used by humans and ideal observers in the companion study (Gosselin \& Schyns, 2001). The features that were used reliably above chance by pigeons were correlated with the features that were used reliably above chance by humans and ideal observers for the emotion task $(r$ pigeons-humans $=.60, p<$ $.05 ; r$ pigeons-ideal $=.47, p<.05)$ and, to a lesser extent, for the gender task $\left(r_{\text {pigeons-humans }}=.20, p<.05 ; r_{\text {pigeons-ideal }}=.12\right.$, $p<.05)$. Note that the pigeons tended to use features near the chin when discriminating gender, but that neither the ideal nor human observer did so. Overall, the pigeons' behavior consistently correlated more highly with the behavior of human observers than with that of ideal observers.

\section{Discussion}

The primary goal of our experiment was to examine the utility of the Bubbles method with pigeons through the use of stimuli and procedures that closely matched those of a companion study with people and computer ideal observers (Gosselin \& Schyns, 2001; Experiment 1). For both emotion and face discrimination tasks, it was entirely possible for our pigeons to have discriminated the training images and for the Bubbles method to have failed to detect any systematic features that the birds used in their discriminations. In such a case, the diagnostic images would have been almost completely gray or would have revealed incidental parts of the stimuli that did not include the most salient features of the faces. However, the Bubbles procedure did not fail to reveal reliable-use salient facial features by pigeons.

An ideal observer was programmed to isolate the features of the faces that contained the most information between happy and neutral categories; human observers also made the same discrimination. The results indicated that much of the control by ideal and human observers discriminating between happy and neutral facial expressions came from features associated with the mouth (see Figure 2). The present Bubbles analysis indicated that the pigeons reliably used features around the mouth to discriminate between happy and neutral facial expressions.

The results from ideal and human observers further indicated that a different set of features should be used to make the gender discrimination; these features include regions around the eyes and the mouth. Bubbles testing indicated that our pigeons did use local features around the eyes, but they ignored regions around the mouth. Unlike ideal and human observers, pigeons did use features near the chin. This disparity does not detract from the general utility of the Bubbles method, but it indicates that different observers may use different features. Overall, the results of this initial test further validate the Bubbles procedure and extend its applicability to the behavior of nonhuman animals.

The correlations in feature use between pigeons and humans for the gender task - although statistically reliable-were smaller than those for the emotion task, perhaps because the features that were used to discriminate gender were more variable than those used to discriminate emotion with these images. Indeed, the correlations among individual human participants in the companion study were smaller for the gender task than the emotion task, when either features that were used reliably above chance or the entire wealth of face information were considered.

Notably, the correlations between pigeons and humans were reliably greater than those between pigeons and ideal observers for both the emotion and gender tasks. It is interesting to note that the diagnostic images for human observers were somewhat more homogeneous than those for pigeons or ideal observers (fewer black areas in Figure 2). Perhaps humans' extensive experience in discriminating faces or differences in the proportion of brain tissue devoted to processing faces accounts for such disparities. Nonetheless, these results suggest that pigeons use face information much as humans do, even though neither species may optimally use the most informative regions of human faces (indicated by the ideal observer) to solve the two tasks.

These results are consistent with other research that has found comparable preferences for face information by humans and nonhuman animals. Ghirlanda, Jansson, and Enquist (2002) trained chickens and university students on a gender discrimination task. During subsequent testing, chickens showed preferences for faces that were somewhat consistent with students' preferences. The results of both Ghirlanda et al. (2002) and the current study suggest that the diagnostic information underlying human face discriminations can be extracted by nonhuman organisms that most likely do not have specialized neural systems (e.g., Kanwisher, McDermott, \& Chun, 1997) for processing faces.

We used the Bubbles procedure in the current experiment to isolate the features of complex visual stimuli-in this case, twodimensional (2-D) gray-level intensities representing human faces-that controlled pigeons' discriminative response. As a first test of the Bubbles procedure with nonhuman animals, we aimed to compare pigeons with humans given the same information space, by using Gosselin and Schyns (2001) previously acquired data.. The Bubbles procedure is not restricted to the assessment of features, however; the procedure can also be used to isolate any feature (e.g., spatial scale, color) of a stimulus space that is associated with discriminative control. In fact, choosing a proper stimulus generation space is one of the most important decisions one makes when setting up a Bubbles experiment (Gosselin \& Schyns, 2002, 2004). Bubbles has been used to isolate a wide variety of stimuli, including 2-D images that have differing spatial scales (Gosselin \& Schyns, 2001; Schyns et al., 2002), a translation-invariant one-dimensional scale space (McCotter, Gosselin, Sowden, \& Schyns, in press; Schyns et al., 2002), and a 3-D space comprising the standard 2-D image plane and time (Vinette, Gosselin, \& Schyns, 2004).

Several different procedures have been used with nonhuman animals to isolate the features of stimuli that control discriminative response. Jitsumori and Yoshihara (1997) trained pigeons to distinguish happy human faces from angry human faces. During later testing, these investigators altered or removed large-scale features of the face such as the eyes, eyebrows, and mouth while they monitored the pigeons' choices to the transformed stimuli. Their results indicated that the eyes and mouth primarily controlled discriminative response.

One apparent advantage of the transformational approach is that the researcher can present combinations of different features to see how different configurations of features combine or compete for control of behavior. Such an approach can also be used with the Bubbles method, but it has not yet been implemented. One disad- 
vantage of the transformational approach is that the researcher must have some advance idea as to what features (shape or otherwise) may control discriminative response. In contrast, the Bubbles procedure has no such restriction.

In another set of studies Troje, Huber, Loidolt, Aust, and Fieder (2000) found that pigeons were able to discriminate between male and female faces. Huber, Troje, Loidolt, Aust, and Grass (2000) used principle-components analysis (PCA) — a multivariate statistical method-to reduce the possible dimensions that could have controlled discriminative response within the stimulus space. Testing images were created on the basis of the dimensions that accounted for the highest proportion of variance. The results suggested that the overall intensity of the face primarily controlled pigeons' behavior. The correspondence-based space of Huber et al. (2000) is a useful technique because it represents the 3-D structure of a face with all textural information mapped onto it: One can identify which aspects of 3-D structure matter for face recognition.

Could global stimulus intensity have been used by pigeons to discriminate the facial images in the current study? Because the Bubbles analysis isolated some local areas of control in the faces for our pigeon observers, and because these areas overlapped with those used by human observers, it is highly likely that local features-much more than global ones-were used with the current stimulus space. In fact, this possibly highlights the main difference between Bubbles and the approaches of both Huber et al. (2000) and Jitsumori and Yoshihara (1997). Although all three of these approaches can be used to search for response-triggering information in the stimulus set that will lead to successful discrimination, one difference among them is how biased they are. In Huber et al. (2000), for example, PCA is used to drastically reduce the dimensionality of the search space in order to predetermine which components may be controlling behavior; the face stimuli are then manipulated accordingly during testing. However, the PCA technique could extract dimensions with little correspondence to the dimensions actually used by human or nonhuman animals. This bias could seriously jeopardize the generality of the results of the experiments done through the use of this correspondence-based space. In contrast, the Bubbles method assesses stimulus control while sampling a stimulus generation space that is as unbiased as possible (here a 2-D image space) and then extracts the critical features of stimulus control. This feature of the Bubbles method also allows one to perform nonlinear analyses of any sort on the data (Gosselin \& Schyns, 2004; Schyns, et al., 2002).

Another technique that has recently been used in the animal literature to assess the use of visual stimulus information is reverse correlation. Martin-Malivel, Mangini, Biederman, and Fagot (2002) used reverse correlation to see what features baboons used to discriminate human from baboon faces. The baboons discriminated between human-baboon morphed images that contained random background noise and made the images appear more like a human on some trials and more like a baboon on other trials. The difference between the patterns of noise for each type of categorization indicated the critical information that was controlling discriminative response. It has been demonstrated that reverse correlation and Bubbles are somewhat complementary techniques (Gosselin, \& Schyns, 2002, 2004; Murray \& Gold, 2004). The latter reveals the information represented in memory, whereas the former reveals the intersection between the represented and the available information (as in the studies of Huber et al., 2000, and Jitsumori \& Yoshihara, 1997).

It appears that Bubbles is a powerful tool that has far-reaching use, particularly when one is attempting to isolate the fine-grained features of complex natural stimuli that may control visual discrimination performance. Although Bubbles appears to be especially useful with naturalistic stimuli, it may also be useful in identifying features of artificial stimuli that may be predicted to be diagnostic on theoretical grounds (e.g., geons; Biederman, 1987). Thus, although we have documented the usefulness of the Bubbles procedure in the present study with human faces as testing images, Bubbles would also appear to be well-suited to exploring stimulus control with a wide variety of natural and artificial stimuli.

\section{References}

Bhatt, R. S., Wasserman, E. A., Reynolds, W. F., Jr., \& Knauss, K. S. (1988). Conceptual behavior in pigeons: Categorization of both familiar and novel examples from four classes of natural and artificial stimuli. Journal of Experimental Psychology: Animal Behavior Processes, 14, 219-234.

Biederman, I. (1987). Recognition-by-components: A theory of human image understanding. Psychological Review, 94, 115-147.

Ghirlanda, S., Jansson, L., \& Enquist, M. (2002). Chickens prefer beautiful humans. Human Nature, 13, 383-389.

Gibson, B. M., \& Wasserman, E. A. (2003). Pigeons learn stimulus identity and stimulus relations when both serve as redundant, relevant cues during same-different discrimination training. Journal of Experimental Psychology: Animal Behavior Processes, 29, 84-91.

Gosselin, F., \& Schyns, P. G. (2001). Bubbles: A technique to reveal the use of information in recognition tasks. Vision Research, 41, 2261-2271.

Gosselin, F., \& Schyns, P. G. (2002). RAP: A new framework for visual categorization. Trends in Cognitive Science, 6, 70-77.

Gosselin, F., \& Schyns, P. G. (2004). No trouble with bubbles: A reply to Murry and Gold. Vision Research, 44, 471-477.

Herrnstein, R. J. (1990). Levels of stimulus control: A functional approach. Cognition, 37, 133-166.

Herrnstein, R. J., Loveland, D. H., \& Cable, C. (1976). Natural concepts in pigeons. Journal of Experimental Psychology: Animal Behavior Processes, 2, 285-311.

Huber, L., \& Lenz, R. (1993). A test of the linear feature model of polymorphous concept discrimination with pigeons. Quarterly Journal of Experimental Psychology: Comparative and Physiological Psychology, 46B, 1-18.

Huber, L., Troje, N. F., Loidolt, M., Aust, U., \& Grass, D. (2000). Natural categorization through multiple feature learning in pigeons. Quarterly Journal of Experimental Psychology: Comparative and Physiological Psychology, 53B, 341-357.

Jitsumori, M., \& Yoshihara, M. (1997). Categorical discrimination of human facial expressions by pigeons: A test of the linear feature model. Quarterly Journal of Experimental Psychology: Comparative and Physiological Psychology, 50B, 253-268.

Kanwisher, N., McDermott, J., \& Chun, M. M. (1997). The fusiform face area: A module in human extrastriate cortex specialized for face perception. Journal of Neuroscience, 17, 4302-4311.

Lea, S. E. G., Lohmann, A., \& Ryan, C. M. E. (1993). Discrimination of five-dimensional stimuli by pigeons: Limitations of feature analysis. Quarterly Journal of Experimental Psychology: Comparative and Physiological Psychology, 46B, 19-42.

Mackintosh, N. J. (1995). Categorization by people and by pigeons: The twenty-second Bartlett memorial lecture. Quarterly Journal of Experimental Psychology: Comparative and Physiological Psychology, 43B, 297-322. 
Martin-Malivel, J., Mangini, M., Biederman, I., \& Fagot, J. (2002, March). Human-Baboon discrimination and representation by humans and baboons (Papio papio). Paper presented at the annual meeting of the Comparative Cognition Society, Melbourne, FL.

McCotter, M., Gosselin, F., Sowden, P. \& Schyns, P. G. (in press). The use of visual information in natural scenes categorization. Visual Cognition.

Murray, R. F., \& Gold, J. M. (2004). Trouble with bubbles. Vision Research, 44, 461-470.

Oliva, A., \& Schyns, P. G. (2000). Colored diagnostic blobs mediate scene recognition. Cognitive Psychology, 41, 176-210.

Schyns, P. G., Bonnar, L., \& Gosselin, F. (2002). Show me the features! Understanding recognition from the use of visual information. Psychological Science, 13, 402-409.

Tjan, B. S., Braje, W. L., Legge, G. E., \& Kersten, D. (1995). Human efficiency for recognizing 3-D objects in luminance noise. Vision Research, 35, 3053-3069.
Troje, N. F., Huber, L., Loidolt, M., Aust, U., \& Fieder, M. (2000). Categorical learning in pigeons: The role of texture and shape in complex static stimuli. Vision Research, 39, 353-366.

Vinette, C., Gosselin, F., \& Schyns, P. G. (2004). Spatio-temporal dynamics of face recognition in a flash: It's in the eyes! Cognitive Science, 28, 289-301.

von Ferson, L., \& Lea, S. E. G. (1990). Category discrimination by pigeons using five polymorphous features. Journal of the Experimental Analysis of Behavior, 54, 69-84.

Wasserman, E. A. (1993). Picture perception: A bird's eye view. Current Directions in Psychological Science, 2, 184-189.

Received August 5, 2004

Revision received November 21, 2004

Accepted January 21, 2005 